\title{
Äquivalenzbeziehungen und Divergenz am Beispiel von deutschen Phraseologismen mit Tierbezeichnungen im Komponentenbestand und ihren polnischen Entsprechungen
}

\author{
Hanna Stypa (Bromberg)
}

\begin{abstract}
This paper discusses the results of the research into German animal idioms and their Polish translations. It centres on equivalence, but also addresses the issue of multiple counterparts in translation which we deal with when an idiom in SL is matched by several counterparts in TL. The article starts with the presentation of the methodology behind this contrastive, GermanPolish study and the terms used (with 'equivalence' being the key term). The following part offers an empirical analysis of selected multiple counterparts.
\end{abstract}

\section{$1 \quad$ Einleitung}

Der vorliegende Beitrag basiert auf den Untersuchungen, die im Rahmen eines umfangreicheren Projekts durchgeführt wurden (vgl. Stypa 2014). Sie setzten sich zum Ziel die Äquivalentenermittlung und Analyse der interlingualen Äquivalenzbeziehungen zwischen den deutschen Phraseologismen mit Tierbezeichnungen und ihren Entsprechungen im Polnischen. Der vorliegende Beitrag ist als Darstellung der theoretischen Prämissen und synthetische Zusammenfassung der Untersuchungsergebnisse konzipiert. Das Augenmerk wird dabei auf einen ausgewählten Aspekt gerichtet, und zwar auf die Divergenz.

\section{Phraseologismen - Definition}

Als Phraseologismen werden Einheiten aufgefasst, die sich durch folgende Merkmale auszeichnen:

- Polylexikalität - Phraseologismen bestehen aus mindestens zwei Wörtern, wobei eines von ihnen ein Autosemantikum ist und die lexikalischen Komponenten eine syntaktische Wortgruppe oder einen Satz bilden. ${ }^{1}$

\footnotetext{
${ }^{1}$ Folgende festgeprägte Einheiten, die Satzstruktur aufweisen, werden zum Phraseologiebereich nicht gerechnet: Sprichwörter, Wellerismen, Sentenzen, Maximen, Aphorismen (vgl. Fleischer 1997: 76-82; Burger 2003: 101104, 120-123; Szczęk 2010: 66-71).

Linguistik online 74, 5/15 - http://dx.doi.org/10.13092/lo.74.2228
}

CC by 3.0 
- Stabilität - Phraseologismen gelten als relativ stabile Verbände, d. h. sie lassen Umformungen auf der morphologischen Ebene sowie Veränderungen im lexikalischen Bestand nur in beschränktem $\mathrm{Maß} \mathrm{zu.}{ }^{2}$

- Idiomatizität - Die Gesamtbedeutung der Phraseologismen entsteht im Laufe von Metaphorisierungsprozessen und ist nicht selten kulturhistorisch bedingt, so dass sie sich aus den Bedeutungen der Komponenten nicht ableiten lässt. In Bezug auf dieses Kriterium unterscheidet man voll-, teil- und nichtidiomatische Phraseologismen: Nur ein Teil von Phraseologismen ist semantisch undurchsichtig. Zum Phraseologiebereich gehören auch solche Konstruktionen, deren Bestandteile ihre wörtliche Bedeutung beibehalten haben (vgl. u. a. phraseologische Vergleiche).

- Lexikalisierung und Reproduzierbarkeit - Phraseologismen fungieren als Elemente eines Lexikons. Daraus ergibt sich die Tatsache, dass sie bei der Äußerung nicht jedes Mal neu nach syntaktischen Mustern produziert, sondern als fixierte Einheiten reproduziert werden (vgl. Fleischer 1997: 68; Burger 2003: 11-15).

\section{Korpus und Untersuchungsmethode}

Sowohl das deutsche Korpus als auch die polnischen Äquivalente entstammen lexikographischen Quellen. Die deutschen Einheiten werden aus einsprachigen phraseologischen Wörterbüchern exzerpiert, u. a. DWDI (2002), Schemann (1993), Müller (1994). Die polnischen Konstruktionen werden einsprachigen phraseologischen Lexika, u. a. Müldner-Nieckowski (2003), Kłosińska/Sobol/Stankiewicz (2005), sowie Wörterbüchern der polnischen Gegenwartssprache, u. a. Dunaj (1999), Szymczak (2002), entnommen. Außerdem werden zweisprachige Wörterbücher zu Rate gezogen, u. a. Ehegötz et al. (1989). Bei der Zusammenstellung des sprachlichen Materials im Deutschen werden u. a. folgende Einheiten nicht berücksichtigt: Phraseologismen mit Bezeichnungen für Tiere, die nur in Märchen und Mythen vorkommen, Phraseologismen, die Bezeichnungen für Körperteile der Tiere enthalten, Phraseologismen, die eine adjektivische Tierkomponente beinhalten, Phraseologismen, deren Gebrauch regional beschränkt ist, und Phraseologismen mit unikalen Komponenten.

Der Vergleich hat unilateralen Charakter. Deutsch gilt als Ausgangs-, Polnisch als Zielsprache. Die Methode der Untersuchung ist ein induktives Vorgehen: "Einzelnen phraseologischen Einheiten der Sprache $\mathrm{L}_{1}$ werden einzelne (phraseologische) Einheiten der Sprache $\mathrm{L}_{2}$ gegenübergestellt und beide Entitäten als Glieder einer binären Opposition verglichen" (Hessky 1987: 50). Die Grundlage der Kontrastierung bildet die semantische Äquivalenz. Die Erforschung ist synchron orientiert und auf die Langue-Ebene beschränkt.

\section{$4 \quad$ Kontrastive Analyse und Äquivalenzbegriff}

Phraseologismen sind komplexe sprachliche Phänomene, die formale Vielfalt aufweisen und sich durch komplizierte innere Strukturierung kennzeichnen. Um das Wesen von phraseologischen Einheiten zu erfassen, soll bei ihrer Untersuchung die semantisch-

\footnotetext{
${ }^{2} \mathrm{Zu}$ Varianten und Modifikationen von Phraseologismen vgl. u. a. Barz (1992), Korhonen (1992),Wotjak (1994), Pociask (2007).
} 
lexikalische, morphosyntaktische wie auch stilistisch-pragmatische Dimension einbezogen werden (vgl. u. a. Eckert 1990: 488-491; Eismann 1995: 97; Földes 1996: 17; Korhonen/Wotjak 2001: 228; Laskowski 2003: 132). Somit findet der zwischensprachliche Vergleich auf vier folgenden Stufen statt:

- Gesamtbedeutung des Phraseologismus,

- lexikalische Besetzung der Komponenten,

- Typ des Phraseologismus,

- Morphosyntax.

Zuerst wird die semantische Struktur der Phraseologismen analysiert, die Folgendes umfasst: Kernbedeutung, präzisierende Seme sowie Konnotationen, darunter Stilschicht und die Einstellung des Sprechers, z. B. weiße Mäuse sehen: Kernbedeutung [Wahnvorstellungen haben], präzisierendes Sem, das die allgemeine Kernbedeutung spezifiziert [im Betrunkenenzustand], konnotative Seme: [umgangssprachlich], [scherzhaft]). ${ }^{3}$ Die zweite Stufe der Gegenüberstellung ist die lexikalische Besetzung der Komponenten. Der Analyse wird die Bedeutung der einzelnen Bestandteile von Phraseologismen unterzogen. ${ }^{4}$ Auch die Gliederzahl wird dabei berücksichtigt. Beim Typ des Phraseologismus wird der Konstruktionstyp der deutschen und der polnischen Einheiten bestimmt (verbaler, nominaler, adverbialer Phraseologismus, phraseologischer Vergleich, phraseologisierter Teilsatz, satzwertiger Phraseologismus). Auf der Ebene der Morphosyntax kontrastiert man die morphologisch-syntaktische Struktur der Phraseologismen. Diese wird im Hinblick auf die Wortart der einzelnen Komponenten, ihre morphologischen Kategorien, interne und externe Valenz sowie die syntaktische Funktion im Verband untersucht.

Aus den Kombinationen der Übereinstimmungen und Unterschiede auf allen Stufen der Kontrastierung entsteht ein Netz von Äquivalenztypen und -gruppen. Es werden drei Hauptäquivalenztypen ermittelt: Voll-, Teil- und Nulläquivalenz. ${ }^{5}$ Der Terminus Äquivalenz wird als die semantisch-formale Übereinstimmung sprachlicher Zeichen bzw. Zeichenkombinationen zweier oder mehrerer Sprachen aufgefasst (vgl. Krohn 1994: 73; Laskowski 2003: 129). ${ }^{6}$ In den vorliegenden Untersuchungen wird die Ansicht vertreten, dass Äquivalenz keine völlige Identität, sondern vielmehr eine teilweise Gleichheit ist, die sich graduieren lässt und bei der man über- und untergeordnete Aspekte ermitteln kann. Als die

\footnotetext{
${ }^{3}$ Die semantische Struktur der Phraseologismen wird anhand von synchronen einsprachigen phraseologischen Wörterbüchern erschlossen.

${ }^{4}$ Die Semantik der phraseologischen Komponenten wird vorwiegend anhand von einsprachigen allgemeinen Wörterbüchern ermittelt.

${ }^{5}$ Die Bezeichnungen Voll-, Teil- und Nulläquivalenz verwenden u. a. Krohn (1994), Chrissou (2001), Gondek/Szczęk (2001), Laskowski (2003). In der einschlägigen Literatur sind außer diesen Termini folgende gebräuchlich: absolute/totale/völlige/vollständige Äquivalenz, partielle/relative Äquivalenz und keine Äquivalenz/Nicht-Äquivalenz (vgl. u. a. Sternemann et al. 1983; Hessky 1987; Ehegötz 1990; Eismann 1995; Toledo 2004).

${ }^{6}$ Vgl. dazu u. a. den Ansatz von Sternemann et al. (1983: 43-47), wo die semantische und formale Übereinstimmung getrennt behandelt werden. Als Äquivalenz wird von den Autoren die Übereinstimmung auf der semantischen Ebene bezeichnet. In Bezug auf den morphologisch-syntaktischen Aspekt gebrauchen sie den Begriff Kongruenz.
} 
dominierende Größe gilt in der Analyse die semantische Äquivalenz, was zur Folge hat, dass die Bedeutung der deutschen Phraseologismen zum Ausgangspunkt für die Erschließung von Entsprechungen im Polnischen wird. Dieser Ansatz ermöglicht somit den Vergleich der Phraseologismen mit völlig abweichender Besetzung der Komponenten wie die Kontrastierung von Phraseologismen mit nichtphraseologischen Ausdrücken - vorausgesetzt, dass sie eine (annähernd) gleiche semantische Struktur aufweisen.

Volläquivalenz besteht immer dann, wenn sich die möglichst vollständige Übereinstimmung zwischen den gegenübergestellten Einheiten auf allen vier Ebenen der Untersuchung feststellen lässt. Teiläquivalenz liegt bei einer völligen oder partiellen Gleichheit in der Gesamtbedeutung des Phraseologismus sowie bei unterschiedlich ausgeprägten Übereinstimmungen und Differenzen im Komponentenbestand, im Typ des Phraseologismus und im Bereich der Morphosyntax vor. Sehr treffend äußert sich dazu Ehegötz (1990: 2): „Die partielle oder Teiläquivalenz erweist sich [...] als ein Sammelbecken der unterschiedlichsten Abstufungen von Nicht-Übereinstimmungen in äquivalenzbestimmenden Faktoren, die bis hin $\mathrm{zu}$ einer minimalen Übereinstimmung zwischen Ausgangssprache-Phrasem und Zielsprache-Entsprechung in nur einem Parameter gehen kann“. Als Nulläquivalenz werden alle Fälle interpretiert, in denen die systemhafte Entsprechung im Polnischen nicht vorhanden ist. Dem deutschen Phraseologismus wird dann eine nichtphraseologische Einheit gegenübergestellt, die in formaler Hinsicht völlig verschieden ist. In Bezug auf die semantische Struktur geben solche Pendants die Bedeutungen der deutschen Phraseologismen wieder, was aber den stilistisch-expressiven Aspekt betrifft, gehen diese Qualitäten meistens (bei Paraphrasen und z. T. bei freien Wortverbindungen) verloren.

\section{Untersuchungsergebnisse}

Das deutsche Korpus besteht aus 340 Phraseologismen mit Tierbezeichnungen im Komponentenbestand. Zum Gegenstand der Kontrastierung werden insgesamt 580 Einheitenpaare. Dieser zahlenmäßige Unterschied ergibt sich daraus, dass die Sememe der mehrdeutigen Konstruktionen sowie manche lexikalischen Varianten der Phraseologismen gesondert behandelt werden. Außerdem werden im zusammengestellten Material Fälle der Divergenz ermittelt. Von den analysierten deutsch-polnischen Paaren konnte man

- 45 als volläquivalent $(7,7 \%)$,

- 399 als teiläquivalent $(68,9 \%)$,

- 136 als nulläquivalent $(23,4 \%)$ einstufen.

Ein Teil der volläquivalenten polnischen Entsprechungen erweist sich als Entlehnungen aus dem Deutschen, u. a. tu leży pies pogrzebany von dt. da liegt der Hund begraben, kupować kota $w$ worku von dt. die Katze im Sack kaufen. Es lassen sich Fälle von Interphraseologismen feststellen, u. a. das Trojanische Pferd - koń trojański (griechische Mythologie), das schwarze Schaf - czarna owca (Bibel). Als phraseologische Internationalismen gelten zudem Vergleiche, die auf die Fabeln von Äsop zurückzuführen sind, u. a. stur wie ein Esel - uparty jak osiot, listig wie ein Fuchs - chytry jak lis, kämpfen wie ein Löwe - walczyć jak lew. Ähnliche Lebenserfahrungen, Vorstellungen und metaphorische Denkweise der Sprecher können als Ursache von weiteren Parallelismen angesehen werden, u. a. eine dumme Gans - 
głupia gęś, jmdn. wie einen Hund behandeln - traktować kogoś jak psa, glatt wie ein Aal śliski jak węgorz, stumm wie ein Fisch - niemy jak ryba. Im Rahmen der Teiläquivalenz werden neun Untergruppen ermittelt, die nach dem abnehmenden Grad der Übereinstimmung auf den Untersuchungsebenen geordnet sind. Am zahlreichsten vertreten (123 Fälle, 30,8\% der Gesamtzahl von teiläquivalenten Paaren) ist die Untergruppe mit folgenden Parametern: völlige Gleichheit in der Gesamtbedeutung und im Typ des Phraseologismus sowie Abweichungen auf der Stufe der lexikalischen Besetzung von Komponenten und der Morphosyntax, u. a. schwimmen wie eine bleierne Ente - plywać jak siekiera, ein Heimchen am Herde - kura domowa, \{jmdm.\} die Würmer aus der Nase ziehen - ciagnać \{kogoś\} za język. Die zwei größten Gruppen der nulläquivalenten Pendants stellen Einzellexeme (63 Fälle, 46,3\% der Gesamtzahl von nulläquivalenten Belegen) und Paraphrasen (62 Fälle, $45,6 \%$ der Gesamtzahl von nulläquivalenten Belegen) dar. Vereinzelt entsprechen den deutschen Phraseologismen freie Wortverbindungen und Sprichwörter, u. a. ein kleiner Fisch - płotka (Einzellexem), das hält ja kein Pferd aus - wykrzyknienie wyrażajace, że coś (najczesściej sytuacja lub dane warunki) jest nie do wytrzymania (Paraphrase), ein linker Vogel - podejrzany typ (freie Wortverbindung), mit den Wölfen heulen - kiedy wejdziesz między wrony, musisz krakać jak i one (Sprichwort).

\section{Divergenz}

Mit Divergenz hat man zu tun, wenn eine Einheit in $\mathrm{L}_{1}$ über mehrere Entsprechungen in $\mathrm{L}_{2}$ verfügt (vgl. Wotjak 1992: 44). ${ }^{7}$ Bei der Analyse werden 89 Fälle der Divergenz festgestellt. Die größte Anzahl der polnischen Pendants entspricht drei synonymen Phraseologismen wie vom wilden Affen gebissen, einen Floh im Ohr haben und <eine Meiseleinen Vogel> haben ,nicht recht bei Verstand sein, verrückte Dinge tun, sich unvernünftig benehmen, idiotische Einfälle haben'. Es stehen ihnen jeweils 13 Äquivalente gegenüber: mieć $<$ bzika/hopla/kota/kręćka/fioła/szmergla/świra>, mieć kuku na muniu, mieć nie po kolei w głowie, mieć nie wszystko po kolei, mieć nierówno pod sufitem, nie mieć piatej klepki, \{komuś\} brakuje piątej klepki, nie mieć wszystkich $w$ domu, upaść na głowę, z byka spaść, najeść się <szaleju/blekotu>, <padać/rzucać się> \{komuś\} na mózg, <szajba/palma> $\{k o m u s ́$ \} odbija. Meistens haben die deutschen Einheiten zwei (40 Fälle) oder drei (27 Fälle) Entsprechungen im Polnischen. Im zusammengestellten Korpus liegt Divergenz vorwiegend nur in den Untergruppen der Teiläquivalenz vor (66 Fälle). Viel seltener stehen den deutschen Konstruktionen sowohl voll- als auch teiläquivalente Entsprechungen gegenüber (10 Fälle). Zehn deutsche Einheiten verfügen über mehrere nichtphraseologische Pendants im Polnischen. In drei Fällen werden sowohl teil- als auch nulläquivalente Entsprechungen ermittelt. Im Folgenden werden ausgewählte Beispiele der Divergenz in fünf Gruppen eingeteilt.

\footnotetext{
${ }^{7}$ Bei interlingualen Vergleichen kann auch eine umgekehrte Situation vorkommen, nämlich dass mehrere Einheiten in $\mathrm{L}_{1}$ ein gemeinsames Äquivalent in $\mathrm{L}_{2}$ haben. Diese Erscheinung wird Konvergenz genannt (vgl. Wotjak 1992: 44). Um den Rahmen des vorliegenden Beitrags nicht zu sprengen, wird dieses Phänomen nicht berücksichtigt.
} 


\subsection{Gruppe 1 (10 Fälle)}

Ein Volläquivalent wird durch ein Teiläquivalent begleitet, das sich von der deutschen Einheit nur auf der lexikalischen Ebene unterscheidet. Das Zustandekommen solcher Kombination wird dadurch verursacht, dass die polnischen Entsprechungen in lexikalischen Varianten erscheinen:

(1) stark wie ein Bär

'kräftig sein, in Bezug auf einen Mann'
- $\quad<$ silny/mocny> jak niedźwiedź

- $<$ silny/mocny $>$ jak $<$ tur $/$ byk $>$

Die körperliche Stärke eines Mannes wird in der deutschen Sprache mit einem Bären assoziiert. Im Polnischen bringt man diese Eigenschaft ebenso mit einem Bären (niedźwiedź) wie auch mit einem Auerochsen (tur) und einem Stier (byk) in Verbindung.

\subsection{Gruppe 2 (26 Fälle)}

Alle polnischen Äquivalente eines deutschen Phraseologismus gehören derselben Gruppe der Teiläquivalenz an. Hier lassen sich folgende Tendenzen beobachten:

Die Unterschiede zwischen den kontrastierten Einheiten kommen auf der lexikalischen und morphosyntaktischen Ebene vor:

(2) das Ei unterm Huhn verkaufen müssen

'kein Geld haben'
- cienko przaśść

- nie śmierdzieć groszem

- nie mieć grosza przy duszy

- klepać biedę

Der deutsche Phraseologismus enthält ein Bild aus dem Bereich der ländlich-bäuerlichen Lebensumstände. In Geldnot verkauft man sogar das Ei, das man noch nicht hat, weil es noch nicht ausgereift ist. In cienko przaść wird auf das Spinnen, eine schon in Vergessenheit geratene Tätigkeit, Bezug genommen. Wenn man nicht über ausreichend Geld verfügt, wird nur ein dünnes Garn gesponnen. In nie śmierdzieć groszem und nie mieć grosza przy duszy kommt die Komponente grosz ,Groschen', in klepać biedę dagegen bieda ,Not" vor. Beide Lexeme stellen die direkte Beziehung zur Bedeutung der Einheiten her. Der deutsche Phraseologismus weist folgende Struktur auf: VP $\rightarrow \mathrm{N}_{\text {Nom }}+\mathrm{N}_{\text {Präp-gr(lokal) }}+\mathrm{V}_{\text {Inf }}+\mathrm{V}_{\text {Mod. }}$ Die Struktur der polnischen Entsprechungen ist wie folgt: cienko przaśśc (VP $\rightarrow \mathrm{Adv}+\mathrm{V})$, nie śmierdzieć groszem $\left(\mathrm{VP} \rightarrow \mathrm{Neg}+\mathrm{V}+\mathrm{N}_{\text {Instr }}\right.$ ), nie mieć grosza przy duszy $(\mathrm{VP} \rightarrow \mathrm{Neg}+\mathrm{V}+$

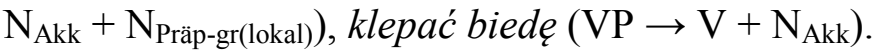

Die Nicht-Übereinstimmungen werden in der Semantik (präzisierende Seme), in der lexikalischen Besetzung der Komponenten sowie in der Morphosyntax festgestellt:

(3) wie das Kaninchen vor der Schlange (stehen)

'handlungsunfähig, starr vor Schrecken'
- stanać jak wryty

- stanać jak stup soli

- stanać jak rażony piorunem

- stanać jakby $\{w$ kogoś\} piorun $<$ strzelit/trafit/razit $>$ 
Verglichen mit der Bedeutung der deutschen Konstruktion ist die semantische Struktur der polnischen Entsprechungen um das Sem [vor Verwunderung] reicher. In den polnischen Einheiten kommen keine Tierbezeichnungen vor. Der im deutschen Phraseologismus ausgedrückte Zustand wird mit anderen Bildern dargestellt, und zwar dass jemand wie angewurzelt dasteht, erstarrt und wie von einem Blitz getroffen wird. Die deutsche Einheit hat folgende Struktur: $\mathrm{PhV} \rightarrow \mathrm{Adv}_{\text {Vergl }}+\mathrm{N}_{\mathrm{Nom}}+\mathrm{N}_{\text {Präp-gr(lokal) }}+(\mathrm{V})$. Die polnischen Pendants weisen dagegen folgende Struktur auf: stanać jak wryty $\left(\mathrm{PhV} \rightarrow \mathrm{V}+\mathrm{Adv}_{\mathrm{Vergl}}+\mathrm{Part}\right.$ II), stanać jak stup soli $\left(\mathrm{PhV} \rightarrow \mathrm{V}+\mathrm{Adv}_{\mathrm{Vergl}}+\mathrm{N}_{\mathrm{Nom}}+\mathrm{N}_{\mathrm{Gen}(\mathrm{Attr})}\right)$, stanać jak rażony piorunem $\left(\mathrm{PhV} \rightarrow \mathrm{V}+\mathrm{Adv}_{\mathrm{Vergl}}+\mathrm{Part} \mathrm{II}+\mathrm{N}_{\mathrm{Instr}}\right)$, stanać jakby $\{w$ kogoś\} piorun $<$ strzelit/trafit/razil $>$ $\left(\mathrm{PhV} \rightarrow \mathrm{V}+\mathrm{AdV}_{\text {Vergl }}+\left\{\mathrm{N}_{\text {Präp-gr }}\right\}+\mathrm{N}_{\mathrm{Nom}}+\mathrm{V}_{\text {Verg }}\right)$.

Die gegenübergestellten Phraseologismen weisen Abweichungen in der Semantik (Stil), auf der lexikalischen und morphosyntaktischen Ebene wie auch im Typ des Phraseologismus auf:

(4) stehlen wie ein Rabe

'stehlen'
- mieć <lepkie/dtugie > <ręce/palce >

- mieć klej na rękach

- $\quad<k l e i c ́ / p r z y k l e j a c ́$ się/lepić się>

$\{k o m u s\}$ do $<$ rąk/ręki $>$

Die deutsche Einheit ist salopp, die polnischen Äquivalente gelten als umgangssprachliche Ausdrücke. Der deutschen Konstruktion liegt die volkstümliche Vorstellung zugrunde, der Rabe neige zum Diebstahl von kleinen (glitzernden) Gegenständen. Die polnischen Pendants bringen dagegen den Grund für die Straftat zum Ausdruck, und zwar handelt es sich um den Klebstoff an den Händen oder Fingern. Außer den Differenzen in der lexikalischen Besetzung der Komponenten weisen die analysierten Einheiten Unterschiede im Typ des Phraseologismus auf. Dem phraseologischen Vergleich im Deutschen stehen verbale Phraseologismen im Polnischen gegenüber. Daraus ergeben sich die NichtÜbereinstimmungen in der Morphosyntax: stehlen wie ein Rabe $\left(\mathrm{PhV} \rightarrow \mathrm{V}+\mathrm{Adv}_{\mathrm{Vergl}}+\right.$ $\left.\mathrm{N}_{\mathrm{Nom}}\right)$, mieć <lepkie/dtugie $><$ ręce/palce $>\left(\mathrm{VP} \rightarrow \mathrm{V}+\mathrm{Adj}_{\mathrm{Attr}}+\mathrm{N}_{\mathrm{Akk}}\right)$, mieć klej na rękach $\left(\mathrm{VP} \rightarrow \mathrm{V}+\mathrm{N}_{\mathrm{Akk}}+\mathrm{N}_{\text {Präp-gr(lokal) }}\right.$ ), <kleić/przyklejać się/lepić się> \{komuś\} do <rąk/ręki> (VP $\rightarrow \mathrm{V}+\left\{\mathrm{N}_{\text {Dat }}\right\}+\mathrm{N}_{\text {Präp-gr(lokal) })}$.

\subsection{Gruppe 3 (40 Fälle)}

Die Entsprechungen eines deutschen Phraseologismus werden unterschiedlichen Gruppen der Teiläquivalenz zugeordnet. Es lassen sich hier folgende Tendenzen ermitteln:

Ein Äquivalent weist nur Unterschiede auf der lexikalischen Ebene auf, das andere (die sonstigen) auch in der Morphosyntax:

(5) schlafen wie ein

$<$ Bär/Dachs/Murmeltier>

'tief schlafen'
- spać jak<suset/kamień>

- spać jak<zabity/zarżnięty>

Im phraseologischen Vergleich des Deutschen findet der lange und tiefe (Winter)schlaf eines Bären, Dachses und Murmeltieres seinen Niederschlag. Im Polnischen assoziiert man diesen Zustand mit dem Schlaf eines Ziesels. Als Comparatum gelten zudem das Substantiv kamień 
,Stein“ und Partizipien von den Verben zabijać ,töten' und zarzynać ,abschlachten'. Dies wirkt sich auf die morphosyntaktische Ebene der Einheiten aus: schlafen wie ein $<$ Bär/Dachs/Murmeltier $>$, spać jak $<$ suset/kamień $>\left(\mathrm{PhV} \rightarrow \mathrm{V}+\mathrm{Adv}_{\mathrm{Vergl}}+\mathrm{N}_{\mathrm{Nom}}\right)$ und spać jak $<$ zabity/zarżnięty $>\left(\mathrm{PhV} \rightarrow \mathrm{V}+\mathrm{Adv}_{\mathrm{Vergl}}+\right.$ Part II $)$.

Ein Äquivalent weist Unterschiede auf der lexikalischen und morphosyntaktischen Ebene auf, das andere (die sonstigen) dagegen auch in der Semantik. Die Abweichungen betreffen die Stilschicht:

(6) sich den Kuckuck $\{<$ um etw./jmdn.>\}

scheren

'sich um jmdn. oder etw. überhaupt nicht

kümmern, jmdn. oder etw. nicht hoch

schätzen, nicht beachten'
- mieć \{coś/kogoś\} w tyłku

- mieć \{coś/kogoś\} w nosie

- mieć \{coś/kogoś\} w gtębokim poważaniu

- $\quad$ mieć $\{c o s ́ / k o g o s ́\}$ gdzieś

- mieć \{coś/kogośs w dupie

In den polnischen Phraseologismen kommen keine Tierbezeichnungen vor. Als Hauptkomponenten fungieren die Substantive tyłek ,Hintern', nos ,Nase', poważanie ,Achtung, Ansehen', dupa ,Arsch'sowie das Adverb gdzieś ,irgendwo'. Der stilistische Wert der Basisbestandteile beeinflusst die Stilschichtzugehörigkeit der ganzen Einheiten. Der deutsche Phraseologismus ist ein salopper Ausdruck, genauso wie das erste polnische Äquivalent. Die drei weiteren sind umgangssprachlich, das letzte derb bzw. vulgär. Die deutsche Konstruktion weist die Struktur VP $\rightarrow \mathrm{N}_{\text {Akk }}+\left\{\mathrm{N}_{\text {Präp-gr }\}}\right\} \mathrm{V}$ auf. Im Folgenden wird die Struktur der polnischen Phraseologismen angegeben: mieć \{coś/kogoś\} w tyłku, mieć

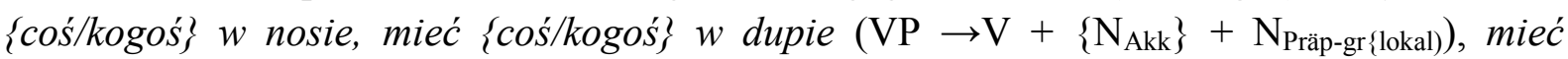
$\left\{\right.$ coś/kogoś\} gdzieś $\left(\mathrm{VP} \rightarrow \mathrm{V}+\left\{\mathrm{N}_{\mathrm{Akk}}\right\}+\mathrm{Adv}\right)$, mieć $\{$ coś/kogośs w glębokim poważaniu (VP

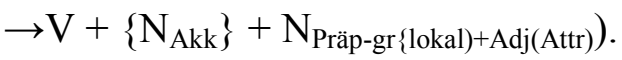

Für ein Äquivalent sind Unterschiede in der Semantik (präzisierende Seme) sowie fehlende Übereinstimmung auf der lexikalischen und morphosyntaktischen Ebene charakteristisch. Beim zweiten (bei den sonstigen) werden noch Abweichungen im Typ des Phraseologismus festgestellt:

(7) ein $<$ rechter/richtiger $>$ Bär

'ein Mann vom großen und kräftigen

Körperbau, z. T. mit langsamen

Bewegungen und innerer Ruhe'

In der semantischen Struktur der Äquivalente fehlt das präzisierende Sem [z. T. mit langsamen Bewegungen und innerer Ruhe]. Die polnischen Phraseologismen enthalten keine Tierbezeichnungen in ihrem Komponentenbestand, als Basisglied dient das Substantiv chłop ,Mann'. Die deutsche Einheit hat die Struktur NP $\rightarrow \operatorname{Adj}_{\text {Attr }}+\mathrm{N}_{\mathrm{Nom}}$, die polnischen haben dagegen: chłop na schwat $\left(\mathrm{NP} \rightarrow \mathrm{N}_{\text {Nom }}+\mathrm{N}_{\text {Präp-gr }}\right)$, chłop jak $<$ dąb/tur $>\left(\mathrm{PhV} \rightarrow \mathrm{N}_{\mathrm{Nom}}+\right.$ $\left.\mathrm{Adv}_{\text {Vergl }}+\mathrm{N}_{\mathrm{Nom}}\right)$. 


\subsection{Gruppe 4 (10 Fälle)}

In diese Gruppe werden Nulläquivalente - Einzellexeme eingestuft. Einige von ihnen stimmen mit den deutschen Phraseologismen semantisch völlig überein:

(8) ein gemeiner Hund

'ein niederträchtiger, skrupelloser und

unehrlicher Mensch, dessen

Verhaltensweise als moralisch schlecht
- drań

- tajdak

- totr

empfunden wird'

Die deutsche Konstruktion und ihre polnischen Entsprechungen sind saloppe, geringschätzige Ausdrücke. Die polnischen Äquivalente lassen sich u. a. mit folgenden deutschen Einzellexemen wiedergeben: Schurke, Lump, Schweinehund.

Ein Teil der polnischen Einzellexeme weicht von den deutschen Phraseologismen in der Stilschicht und/oder in der Einstellung des Sprechers ab:

(9) ein lustiger Vogel

'ein witziger Mensch, der gerne scherzt'
- jajcarz

- zgrywus

Die deutsche Einheit ist umgangssprachlich, die polnischen Lexeme gehören der saloppen Stilschicht an und sind scherzhafte Ausdrücke. Das Substantiv jajcarz steht mit dem Phraseologismus robić sobie \{z kogoś\} jaja,jmdn. täuschen, um ihn zu verspotten, zu veralbern' im Zusammenhang, zgrywus wird vom Verb zgrywać się ,Possen reißen“ abgeleitet.

\subsection{Gruppe 5 (3 Fälle)}

Diese Gruppe umfasst besondere Fälle. Dem deutschen Phraseologismus werden sowohl teilals auch nulläquivalente Pendants gegenübergestellt. Die teiläquivalenten Entsprechungen weisen einen niedrigen Grad der Übereinstimmung auf den Untersuchungsebenen auf, die nichtphraseologischen Einheiten sind Einzellexeme:

(10) eine <Biene/Fliege/Mücke $>$

$<$ machen/drehen $>$

'schnell und (unbemerkt) weggehen,

verschwinden'
- <zniknać/ulotnić się/rozplynać się> jak kamfora

- urwać się

- sptywać

- $\quad$ spadać

- zmywać się

- zwijać się

Der polnische Phraseologismus stimmt nur teilweise in der Bedeutung mit der deutschen Konstruktion überein. Er enthält zusätzlich das Sem [ohne jegliche Spur zu hinterlassen]. Auf den sonstigen Analysestufen lassen sich auch Abweichungen feststellen. Die polnische 
Einheit weist in ihrem Komponentenbestand keine Tierbezeichnung auf. Das schnelle Weggehen einer Person wird damit verglichen, dass sich Kampfer sehr schnell verflüchtigt. Dem verbalen Phraseologismus im Deutschen steht ein Vergleich im Polnischen gegenüber. Daraus resultieren Differenzen auf der morphosyntaktischen Ebene: eine $<$ Biene/Fliege/Mücke $><$ machen/drehen $>\left(\mathrm{VP} \rightarrow \mathrm{N}_{\mathrm{Akk}}+\mathrm{V}\right),<$ zniknąć/ulotnić się/rozplynać się> jak kamfora $\left(\mathrm{PhV} \rightarrow \mathrm{V}+\mathrm{Adv}_{\mathrm{Vergl}}+\mathrm{N}_{\mathrm{Nom}}\right)$. Bei den exzerpierten Einzellexemen handelt es sich um Verben mit mehreren Sememen, darunter gibt es auch das Semem, das der deutsche Phraseologismus aufweist. Die nichtphraseologischen Pendants stimmen mit der deutschen Einheit sowohl in der Denotation als auch in der Konnotation überein.

In diesem Zusammenhang erhebt sich die folgende Frage: Welche Entsprechung hat einen höheren Wert? Die nichtphraseologische, die alle oder fast alle semantischen Inhalte der Einheit der Ausgangssprache wiedergibt, oder ein Phraseologismus, der formal mit dem deutschen nicht übereinstimmt und in semantischer Hinsicht nur partiell adäquat ist? Es wird die Meinung vertreten, dass solche Fragen je nach Fall zu entscheiden sind. Die Aufgabe der kontrastiven Untersuchungen beruht darauf, die möglichen Äquivalente zu ermitteln. Die Entscheidung, welches sich im bestimmten Kontext am besten eignet, liegt bei dem Übersetzer bzw. Dolmetscher.

\section{$7 \quad$ Zusammenfassung}

Die durchgeführte Analyse hat ergeben, dass ca. ein Viertel der deutschen Phraseologismen mit Tierbezeichnungen über mehrere Pendants im Polnischen verfügt. Somit ist Divergenz eine nicht $\mathrm{zu}$ unterschätzende Erscheinung in den kontrastiven Untersuchungen. Divergenz überwiegt deutlich in der Gruppe der Teiläquivalenz. Die meisten polnischen Entsprechungen haben eine völlig andere Komponentenbesetzung und morphosyntaktische Struktur. Einige von ihnen stimmen nur annähernd mit dem deutschen Phraseologismus in semantischer Hinsicht überein - es lassen sich leichte Abweichungen in der Stilschicht oder im Bereich der präzisierenden Seme ermitteln. Von entscheidender Bedeutung für die Ergebnisse der Gegenüberstellung ist die Tatsache, dass in beiden Sprachen unterschiedliche morphosyntaktische Strukturen üblich sind und gleiche Inhalte mit anderen Mitteln ausgedrückt werden.

\section{Literatur}

Barz, Irmhild (1992): "Phraseologische Varianten: Begriff und Probleme". In: Földes, Csaba (ed.) (1992): Deutsche Phraseologie in Sprachsystem und Sprachverwendung. Wien, Edition Praesens: 25-47.

Burger, Harald (2003): Phraseologie: eine Einführung am Beispiel des Deutschen. Berlin: Schmidt.

Chrissou, Marios (2001): "Deutsche und neugriechische Phraseologismen mit animalistischer Lexik. Eine kontrastive Analyse auf der Wörterbuch- und Textebene". EliSe. Essener Linguistische Skripte - elektronisch 1: 89-121. https://www.unidue.de/imperia/md/content/elise/ausgabe_1_2001_chrissou.pdf, [15.12.2011].

DUDEN, Bd. 11 (2002): Redewendungen. Wörterbuch der deutschen Idiomatik. Mannheim etc.: Dudenverlag. (= DWDI)

Dunaj, Bogusław (1999): Słownik współczesnego języka polskiego. Warszawa: Wilga.

ISSN 1615-3014 
Eckert, Rainer (1990): "Spezifisches bei der konfrontativen Untersuchung der Phraseologie zweier oder mehrerer Sprachen". Zeitschrift für Slawistik 35: 488-492.

Ehegötz, Erika (1990): "Versuch einer Typologie von Entsprechungen im zweisprachigen phraseologischen Wörterbuch": 1-6. http://www.fask.unimainz.de/inst/is/polnisch/erika_worbs.html, [14.10.2008].

Ehegötz, Erika et al. (1989): Phraseologisches Wörterbuch Polnisch-Deutsch. Leipzig: Enzyklopaedie.

Eismann, Wolfgang (1995): "Pragmatik und kulturelle Spezifik als Problem der Äquivalenz von Phraseologismen". In: Baur, Rupprecht S./Chlosta, Christoph (1995) (eds.): Von der Einwortmetapher zur Satzmetapher. Akten des Westfälischen Arbeitskreises Phraseologie/Parömiologie. Bochum, Brockmeyer: 95-119.

Fleischer, Wolfgang (1997): Phraseologie der deutschen Gegenwartssprache. Tübingen: Niemeyer.

Földes, Csaba (1996): Deutsche Phraseologie kontrastiv: intra- und interlinguale Zugänge. Heidelberg: Groos.

Gondek, Anna/Szczęk, Joanna (2001): "Farben in den Phraseologismen des Deutschen und des Polnischen". Studia Linguistica XX/2001: 83-94.

Hessky, Regina (1987): Phraseologie. Linguistische Grundfragen und kontrastives Modell deutsch $\rightarrow$ ungarisch. Tübingen: Niemeyer.

Kłosińska, Anna/Sobol, Elżbieta/Stankiewicz Anna (2005): Wielki słownik frazeologiczny z przystowiami. Warszawa: Wydawnictwo Naukowe PWN.

Korhonen, Jarmo (1992): „Morphosyntaktische Variabilität von Verbidiomen“. In: Földes, Csaba (ed.) (1992): Deutsche Phraseologie in Sprachsystem und Sprachverwendung. Wien, Edition Praesens: 49-87.

Korhonen, Jarmo/Wotjak, Barbara (2001): "Kontrastivität in der Phraseologie". In: Helbig, Gerhard et al. (eds.) (2001): Deutsch als Fremdsprache. Ein internationales Handbuch. 1. Halbband. Berlin/New York, de Gruyter: 224-235.

Krohn, Karin (1994): Hand und Fuß. Eine kontrastive Analyse von Phraseologismen im Deutschen und Schwedischen. Göteborg: Acta Universitatis Gothoburgensis.

Laskowski, Marek (2003): Semantische und pragmatische Aspekte der deutschen und polnischen Phraseologie. Zielona Góra: Wydawnictwo Uniwersytetu Zielonogórskiego.

Müldner-Nieckowski, Piotr (2003): Wielki słownik frazeologiczny języka polskiego. Warszawa: Świat Książki.

Müller, Klaus (1994): Lexikon der Redensarten. Gütersloh: Bertelsmann.

Pociask, Janusz (2007): Zu Status und Funktion der idiomatischen Einheit in Pressetexten. Dargestellt an Textbeispielen aus der Neuen Züricher Zeitung. Frankfurt a. M. etc.: Lang.

Schemann, Hans (1993): Deutsche Idiomatik. Die deutschen Redewendungen im Kontext. Stuttgart etc.: Klett.

Sternemann, Reinhard et al. (1983): Einführung in die konfrontative Linguistik. Leipzig: Enzyklopaedie.

Stypa, Hanna (2014): Zoologismen im Deutschen und ihre polnischen Entsprechungen. Bydgoszcz: Wydawnictwo Uniwersytetu Kazimierza Wielkiego.

Szczęk, Joanna (2010): Auf der Suche nach der phraseologischen Motiviertheit im Deutschen (am lexikographischen Material). Dresden etc.: Neisse/Atut. 
Szymczak, Mieczysław (2002): Słownik języka polskiego PWN. Warszawa: Wydawnictwo Naukowe PWN.

Toledo, Emilio (2004): Vergleichende Phraseologie der spanischen und der deutschen Sprache. Untersuchungen anhand einer Auswahl verbaler Phraseologismen mit der allgemeinen Bedeutung "Angriff auf eine Person". Hamburg: Kovač.

Wotjak, Barbara (1992): "Probleme einer konfrontativen Phraseologieforschung am Beispiel verbaler Phraseolexeme". In: Korhonen, Jarmo (ed.) (1992): Untersuchungen zur Phraseologie des Deutschen und anderen Sprachen: einzelsprachspezifisch-kontrastivvergleichend. Akten der internationalen Tagung in Turku. 6.-7.9.1991. Frankfurt a. M. etc., Lang: 39-60.

Wotjak, Barbara (1994): "Fuchs, die hast du ganz gestohlen: Zu auffälligen Vernetzungen von Phraseologismen in der Textsorte Anekdote". In: Sandig, Barbara (ed.) (1994): Europhras 92: Tendenzen der Phraseologieforschung. Bochum, Brockmeyer: 619-650.

\section{Verwendete Symbole}

$\operatorname{Adj}_{\text {Attr }}$

Adv

Adv vergl

$\mathrm{L}_{1} / \mathrm{L}_{2}$

$\mathrm{N}_{\mathrm{Nom} / \mathrm{Gen} / \mathrm{Dat} / \mathrm{Akk} / \text { Instr }}$

$\mathrm{N}_{\text {Gen(Attr) }}$

$\mathrm{N}_{\text {Präp-gr }}$

$\mathrm{N}_{\text {Präp-gr(lokal) }}$

$\mathrm{Neg}$

NP

Part II

$\mathrm{PhV}$

$\mathrm{V}$

$\mathrm{V}_{\text {Inf }}$

$\mathrm{V}_{\text {Mod }}$

$\mathrm{V}_{\text {Verg }}$

VP

$<>$

( )

\{\}
Adjektiv in der Funktion des Attributs

Adverb

Vergleichsadverb

Ausgangssprache/Zielsprache

Substantiv im Nominativ/Genitiv/Dativ/Akkusativ/Instrumental

Substantiv im Genitiv in der Funktion des Attributs

Substantiv als Kern der Präpositionalgruppe

Substantiv als Kern der Präpositionalgruppe in der Funktion der Lokalbestimmung

Negation

nominaler Phraseologismus

Partizip II

phraseologischer Vergleich

Verb

Verb im Infinitiv

Modalverb

Verb im Vergangenheitstempus

verbaler Phraseologismus

in spitzen Klammern stehen austauschbare Komponenten der Phraseologismen

in runden Klammern stehen fakultative Komponenten der Phraseologismen

in geschweiften Klammern stehen Komponenten der Phraseologismen, 
Hanna Stypa: Äquivalenzbeziehungen und Divergenz am Beispiel von deutschen Phraseologismen

die im Kontext besetzt werden

[ ] in eckigen Klammern wird die semantische Struktur der Phraseologismen angegeben 